\title{
Rayonnement acoustique de structures vibrantes : applications au domaine audible
}

\author{
Acoustic radiation of vibrating structures : \\ application to the audible frequencies
}

\author{
par D. Habault
}

C.N.R.S.

Laboratoire de Mécanique et d'Acoustique

The fluid/structure interaction phenomena in Acoustics correspond to the sound radiation of a vibrating structure immersed in a fluid. The vibrations of the structure generate a perturbation of the pressure in the fluid and then the propagation of a signal. The many applications of this theme include Transportation Noise as well as Music. This paper presents some aspects of the numerical simulation of these phenomena along with some examples.

\section{I $\square$ INTRODUCTION}

Le rayonnement acoustique de structures vibrantes, également appelé Vibro-Acoustique, est un domaine qui a pris une importance croissante avec la lutte contre les nuisances sonores et vibratoires dans l'environnement.

Les applications concernent de nombreux domaines industriels, notamment :

- les "Bruits de transports" qui est l'un des secteurs les plus actifs actuellement car la demande est forte, que ce soit en bruit interne (confort des habitacles de véhicules,...), qu'en bruit externe (confort des riverains pour les véhicules terrestres et aériens, furtivité pour les véhicules militaires aériens ou sous-marins,...) ;

- les "Bruits de machines industrielles" ;

- le secteur du Bâtiment avec le confort des habitations (transparence des cloisons,...).

Dans un registre plus agréable à l'oreille, la Musique est également, avec le rayonnement des instruments (timbale, piano,...), un domaine d'applications non négligeable.

Le but des études est généralement la prédiction et la réduction des niveaux de bruit émis par la structure, ce qui nécessite une meilleure compréhension des phénomènes de couplage entre fluide et structure. Actuellement de nombreuses méthodes de prédiction sont développées et de nombreux logiciels sont disponibles.

Le présent article expose quelques aspects de la simulation numérique de ces phénomènes, illustrés par des exemples. Une description de la recherche en France dans ce domaine se trouve dans les Actes publiés lors des Colloques annuels du Groupe de Recherche Vibro-Acoustique du C.N.R.S. [1]. Pour une description des modélisations et méthodes, lire $[2,3,4]$ par exemple.

\section{II — MODÉLISATION DE LA STRUCTURE ET DU FLUIDE}

La modélisation des problèmes de Vibro-Acoustique fait naturellement appel aux disciplines voisines telles que la Mécanique des Fluides, la Mécanique des Matériaux, l'Aérodynamique, etc. La spécificité des problèmes de VibroAcoustique est le couplage entre structure vibrante et fluide. Les inconnues sont la vitesse vibratoire de la structure et la pression acoustique dans le fluide. Généralement, le couplage est modélisé mathématiquement par une condition de continuité des vitesses normales à l'interface structure / fluide.

\subsection{La structure}

La structure peut être simple (plaque, coque) ou complexe (voiture, machine et son capotage, violon,....). Elle est excitée mécaniquement par une force, un moment, etc. Lorsque la structure est excitée en basse fréquence, sa courbe de réponse (déplacement, vitesse,...) en fonction de la fréquence fait apparaître une suite de pics correspondant aux fréquences de résonance du système. Leur emplacement dépend des caractéristiques géométriques et mécaniques de la structure ainsi que du couplage avec le fluide. A ces fréquences particulières, sont associées les déformées du système, appelées modes. La densité de modes croît en fonction de la fréquence. 


\subsection{Le fluide}

\subsubsection{Fluide au repos}

Le fluide est, bien sûr, compressible et, généralement, homogène. L'influence du fluide sur les vibrations de la structure dépend du rapport des masses volumiques du fluide et de la structure. Si le fluide est "léger" (air), l'influence du fluide ("effet de masse ajoutée") est faible et il est possible de développer des approximations "fluide léger" [5]. Si le fluide est lourd (eau), l'influence est non négligeable.

\subsubsection{Fluide en écoulement}

Il faut a priori considérer le problème couplé complet : mouvement du fluide (équations de Navier-Stokes) et mouvement de la structure (équation en vibrations). La résolution du système est alors très lourde. Cependant, dans de nombreux cas, la modélisation peut être simplifiée si l'on suppose que le mouvement de la structure n'a qu'une influence négligeable sur celui du fluide. Les vibrations de la structure sont alors découplées du mouvement du fluide. Dans le cas d'un écoulement turbulent, l'effet de l'écoulement est introduit dans le terme d'excitation sous forme de champ de pression pariétale.

\section{III — MÉTHODES DE PRÉDICTION}

La grande majorité des méthodes a été développée dans le domaine fréquentiel. Les études en domaine temporel sont moins nombreuses mais leur nombre croît depuis quelques années.

\section{- 3.1 Problèmes déterministes et domaine fréquentiel}

Dans le domaine fréquentiel, le choix des méthodes dépend en premier lieu des bandes de fréquence étudiées. L'un des principaux critères de choix d'une méthode est lié à la densité modale dans la bande de fréquence. En basse fréquence, les courbes de pression et de déplacement sont caractérisées par une succession de pics distincts. On utilise classiquement des développements en séries de modes ou des méthodes du type équations intégrales / éléments finis. A plus haute fréquence, la densité de modes croît et les méthodes sont basées sur des approximations de type énergétique.

\subsection{Basses fréquences}

Les principales méthodes sont :

- Développements des fonctions inconnues en série de modes, modes de la structure dans le vide [2] ou modes de la structure couplée au fluide. Ces derniers sont mieux adap-

tés et prennent en compte naturellement un amortissement dû au rayonnement dans le fluide, mais ils sont plus difficiles à calculer. Les modes de la structure dans le vide sont connus analytiquement pour des structures très simples (poutres et plaques minces) et certains types de conditions aux limites.

- Equations intégrales : la connaissance de fonctions de Green (solutions élémentaires en espace infini) permet de représenter déplacement et pression sous formes d'intégrales sur la frontière fluide/structure. Le système d'équations intégrales à résoudre sur la surface de la structure est résolu numériquement à l'aide d'éléments finis de frontière. La fonction de Green pour le fluide (infini ou semi-infini) est bien connue, il n'en est pas de même pour la structure sauf pour des formes canoniques (poutres et plaques minces). La figure 1 présente, pour une plaque raidie, un exemple de comparaison entre niveaux rayonnés calculés et mesurés (fonction de transfert entre deux microphones), moyennés par tiers d'octaves [6]. La plaque $(1 \mathrm{~m} \times 1,54 \mathrm{~m})$ est encastrée dans un baffle rigide et munie de deux raidisseurs.

- Eléments finis : la méthode est basée sur une formulation variationnelle et un maillage des deux domaines de propagation (le fluide et la structure). Elle permet de traiter a priori une structure aussi compliquée soit-elle. Les limitations sont surtout d'ordre numérique (taille du système linéaire à résoudre). Cette méthode pose aussi le problème du maillage d'un domaine s'étendant jusqu'à l'infini. De nombreux types d'éléments finis sont étudiés pour décrire les structures (voir [7] par exemple).

- Méthode couplée éléments finis / équations intégrales : elle consiste à exprimer la pression acoutisque sous forme intégrale à l'aide de la fonction de Green du fluide et à utiliser un maillage "éléments finis de volume" pour la structure. C'est la plus couramment utilisée.

Ces méthodes d'équations intégrales et d'éléments finis sont efficaces surtout en basses fréquences car les dimensions des maillages doivent être proportionnelles aux longueurs d'onde. Les tailles des systèmes à résoudre croissent donc avec la fréquence du phénomène étudié.

\subsubsection{Hautes fréquences}

Dans le domaine des hautes fréquences, les méthodes sont basées sur des bilans d'énergie, évaluant les niveaux d'énergie moyens rayonnés par bandes de fréquence. La principale méthode est la "Statistical Energy Analysis" (S.E.A.) [8] mais d'autres approximations ou méthodes simplifiées sont également mises en œuvre.

La structure est généralement décomposée en sous-systèmes. Un exemple typique d'ap-

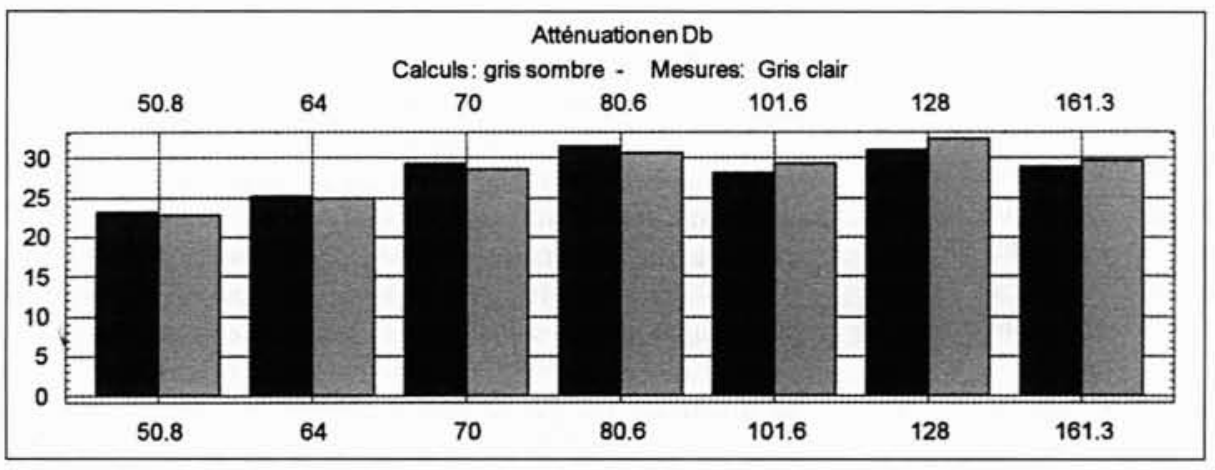

1. Plaque raidie. Fonction de transfert entre deux microphones. Moyennes en tiers d'octave. plications est le cas d'un assemblage de plaques, représentant par exemple les cloisons d'une salle, le but est alors de donner les niveaux d'énergie transmis ou rayonnés par les cloisons. Les principales hypothèses sont :

- forte densité de modes dans la bande de fréquence étudiée,

- faible couplage entre les soussystèmes.

- En S.E.A., on suppose que les 
modes n'interagissent pas. Les couplages entre les sous-systèmes sont pris en compte par des coefficients de couplage dont l'expression dépend du type d'élément. C'est une méthode actuellement très appliquée mais qui demande une grande pratique d'utilisation. Chaque cas nouvellement étudié nécessite un apprentissage.

- La Méthode Energétique Simplifiée (M.E.S.) [1] repose sur des hypothèses similaires et elle néglige les ondes évanescentes et les interférences entre les ondes propagatives.

- D'autres techniques plus approchées

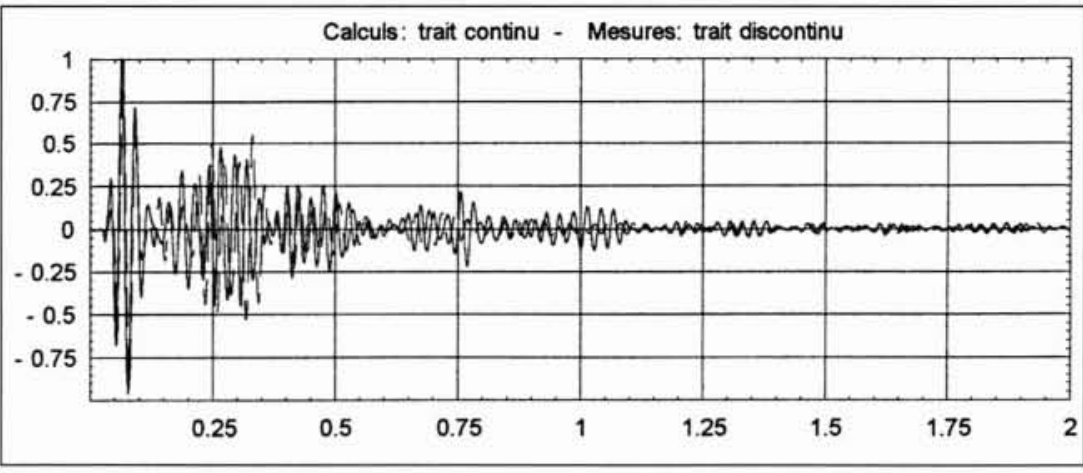

3. Coque "Line2" en eau . Signal rayonné.

de rayonnement pour estimer la puissance acoustique rayonnée par une structure à partir de mesures de vitesses sur la surface vibrante. Le facteur de rayonnement d'une structure est défini comme le rapport de la puissance rayonnée sur la valeur quadratique moyenne de la vitesse vibratoire. Les facteurs de rayonnement sont évalués expérimentalement ou à partir d'expressions analytiques connues pour des géométries simples (plaques). C'est également une technique qui demande un apprentissage.

\subsection{Problèmes déterministes et domaine temporel}

Les représentations temporelles sont plus intéressantes dans le cas de bruits transitoires, d'excitations aléatoires, etc.
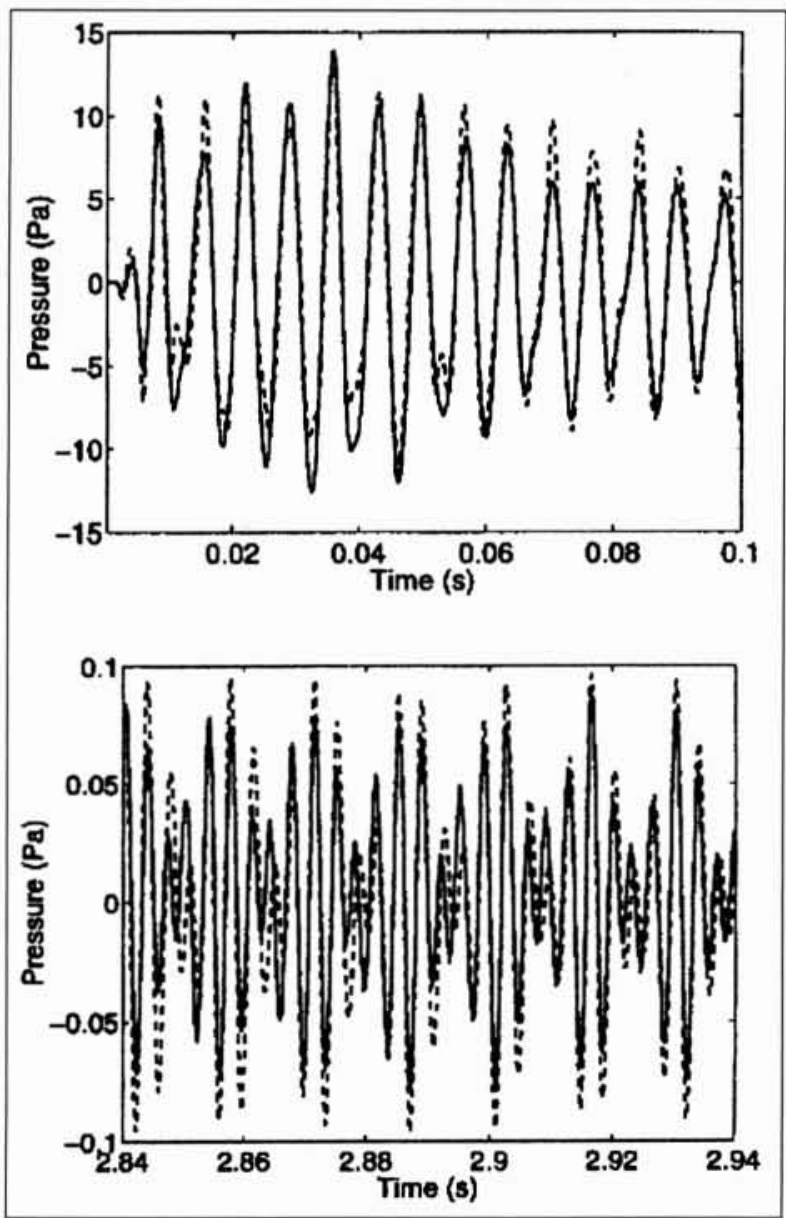

2. Timbale. Pression acoustique rayonnée. Comparaison $\mathrm{calcul} / \mathrm{mesures}$.
Il est alors toujours possible de résoudre en fréquence puis utiliser une transformée de Fourier numérique en temps. Il existe peu de méthodes directement temporelles, telles que celle des potentiels retardés. Les deux exemples présentés ici sont traités directement en domaine temporel et basés sur une formulation variationnelle du problème.

Le premier exemple utilise une méthode d'éléments finis en espace et de différences finies en temps [9]. Il s'agit de décrire le rayonnement en fonction du temps d'une timbale frappée par un maillet. La timbale est modélisée par une membrane tendue sur une cavité ellipsoïdale. La résolution numérique permet de visualiser, en fonction du temps, le déplacement de la membrane et le rayonnement sonore à l'intérieur et à l'extérieur de la cavité. La figure 2 présente une comparaison entre pression acoustique mesurée (trait continu) et calculée (tirets) pendant les 100 premières ms, puis les vibrations libres $2,84 \mathrm{~s}$ après l'impact.

Le second exemple utilise un développement des fonctions inconnues en série de modes de résonance de la structure couplée avec le fluide [6]. Il s'agit d'une coque cylindrique mince fermée par deux hémisphères. Les modes de résonance sont obtenus en résolvant un problème en domaine fréquentiel, le passage au domaine temporel se fait alors analytiquement par transformée de Fourier. La figure 3 représente le signal émis par la coque excitée par un champ incident acoustique, pour une comparaison entre calculs et mesures dans une cuve aquatique. Cet exemple correspond à un problème de diffusion acoustique par des cibles, qui est tout à fait similaire aux problèmes de rayonnement. La différence réside dans le type d'excitation (acoustique / mécanique). Les mêmes méthodes sont applicables. Les problèmes de diffusion acoustique ont pour application principale la détection acoustique de cibles.

\subsection{Problèmes aléatoires}

L'aspect aléatoire peut être dû, par exemple, au type d'excitation. Les inconnues du problème sont alors les densités spectrales de vitesse vibratoire et de pression acoustique.

Dans le cas d'un écoulement turbulent, l'excitation est introduite sous forme d'un champ de pression pariétale. La difficulté est de bien modéliser ce champ. L'exemple cité ici a été réalisé à l'E.C.L. [10] dans le cas d'un écoulement aérodynamique, turbulent, pleinement développé, à l'intérieur d'une coque rigide. La première partie de l'expérimentation avait pour but de caractériser l'écoulement. Des microphones placés à la surface de la coque, répartis dans l'axe de la coque et sur une circonférence, permettent de déduire les densités spectrales de puissance caractérisant l'écoulement et 


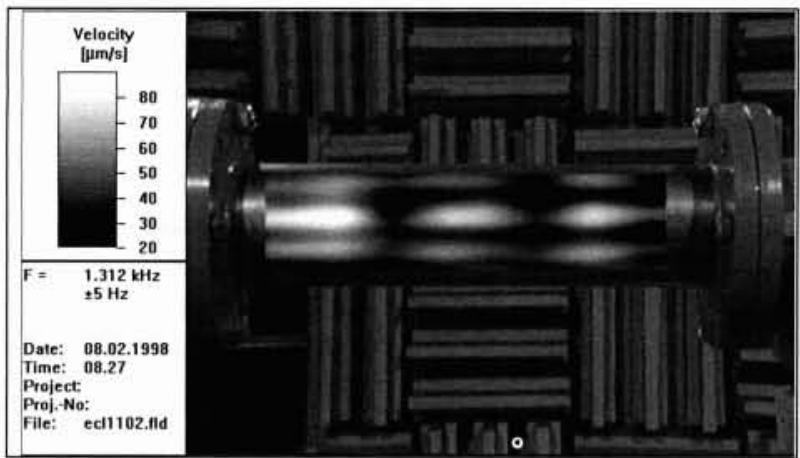

4. Réponse vibratoire d'une coque cylindrique excitée par un écoulement turbulent.

ce, pour des vitesses allant de 60 à $140 \mathrm{~m} / \mathrm{s}$. L'écoulement est modélisé par un modèle de Corcos, pour lequel on détermine des longueurs de corrélation axiale et circonférentielle. La vitesse de convection est déduite de la mesure de la phase de l'interspectre entre deux microphones axiaux. Les mesures à basse fréquence sont rendues difficiles par la présence de phénomènes acoustiques.

Dans la deuxième étape, un tronçon de coque rigide a été remplacé par une coque mince vibrante, de même diamètre. Des mesures, réalisées à l'aide d'un vibromètre laser et de microphones, ont permis de visualiser la vitesse à la surface de la coque et le rayonnement acoustique à l'extérieur de la coque. La figure 4 présente un exemple de réponse vibratoire de la coque à une fréquence de résonance. Les mesures sont en bon accord avec la simulation numérique effectuée au L.M.A. [10]

\section{IV $\square$ PROLONGEMENT DES APPLICATIONS}

La Vibro-Acoustique, en dehors de ses applications directes, a de nombreux prolongements dans d'autres domaines tels que le contrôle actif et la qualité des bruits.

\subsection{Contrôle Actif de Bruit et Vibrations}

Cette technique consiste à modifier, à l'aide de sources vibratoires "secondaires", les vibrations d'une structure afin de diminuer les niveaux de bruit rayonnés. Le domaine d'applications est celui des basses fréquences, pour lequel le champ de pression peut être décrit par quelques modes. Il est donc complémentaire du domaine d'utilisation des matériaux absorbants qui, eux, sont efficaces à plus hautes fréquences. L'article [11] décrit une expérimentation d'atténuation active réalisée dans une chambre anéchoïque du L.M.A., sur une large bande de fréquence. La structure est une plaque mince de dimensions $(0,70 \mathrm{~m} \times 0,54 \mathrm{~m})$, encastrée dans un baffle rigide.

\subsection{Qualité Subjective des Bruits}

La simple réduction de niveaux de bruit, aux fréquences correspondant aux maximums des spectres de rayonnement, n'est pas toujours la meilleure solution. Le bruit ainsi obtenu peut en effet s'avérer plus désagréable que le bruit initial.

Cette constatation a conduit à prendre en compte des critères "subjectifs", car liés aux individus, caractérisant un bruit émis [12]. De nombreuses études sont actuellement développées dans l'industrie, pour transformer le bruit dû à une machine, une voiture, etc. en un bruit moins déplaisant, voire agréable. Il existe des logiciels évaluant ces critères



5. Courbe de sonie (en Barks) pour un signal rayonné par une plaque bafflée.

(sonie, rugosité, acuité,...) et ils peuvent être appliqués aux bruits rayonnés par des structures vibrantes. La figure 5 présente un exemple de courbe de sonie dans le cas d'un signal rayonné par une plaque bafflée excitée mécaniquement.

\section{$V \square$ CONCLUSION}

Cet article a présenté quelques aspects de la modélisation en Vibro-Acoustique qui est actuellement un domaine très actif. Les applications sont nombreuses, la plupart répondent à la forte demande d'un environnement plus silencieux.

Pour résumer très brièvement, les besoins actuels en méthodes de prédiction concernent surtout les moyennes et hautes fréquences, la prise en compte des structures de grandes dimensions (méthodes de sous-structuration,...), la modélisation d'écoulement, etc. Il existe également un besoin de validation des logiciels, notamment sur des cas non-académiques.

\section{BIBLIOGRAPHIE}

[1] Colloques du Groupe de Recherche "Vibro-Acoustique" du C.N.R.S Publications du L.M.A. Marseille. Années 1994, 1995, 1996, 1997, 1998.

[2] C. Lesueur, "Rayonnement acoustique des structures", Collection de la Direction des Etudes et Recherches E.d.F., vol. 66, Eyrolles, Paris 1988.

[3] D. Habault (ed.), "Fluid-Structure Interactions in Acoustics", C.I.S.M Lecture Notes $n^{\circ} 396$, Springer-Verlag. Vienne, parution en 1999.

[4] R. ОнауоN, C. Solze, "Structural Acoustics and Vibration", Academic Press, San Diego, 1998.

[5] D. HaBault, P. Fil.mpl, "Light fluid approximation for sound radiation and diffraction by thin elastic plates". Journal of Sound and Vibration. 213(2), p.333-374, 1998

[6] C. MAURY, "Rayonnement acoustique de structures vibrantes : étude en régime temporel et fréquentiel". Thèse de Docteur-Ingénieur, Université de la Méditerranée, 21 sept. 1999

[7] J.L. Batoz, G. Dhatr, "Modélisation des structures par éléments finis", 3 volumes, Hermès, Paris, 1995.

[8] R. Lyon, "S.E.A. of Dynamical Systems", M.I.T. Press, 1975.

[9] L. Rhaoutt, A. Chaigne, P. Joly, "Time-domain modeling and numerical simulation of a kettledrum", Journal of the Acoustical Society of America, 105(6), p.3545-3562, 1999.

[10] C. Durant, G. Robert, P. Filippl, P.O. Mattel, "Vibro-acoustic response of a thin cylindrical shell excited by a turbulent internal flow...", soumis au Journal of Sound and Vibration.

[11] E. Friot, L. Deschaintres, "Feedback control of the transmission los through a plate with piezo devices". Movic 98, Fourth International Conference on Motion and Vibration Control, Zurich, p.665-670, Ed. G Schweitzer, R. Siegwart and P. Cattin, 25-28 août 1998.

[12] Journée d'information "Les nouveaux outils de la qualité acoustique", 22 Octobre 1996, Publications du Centre Technique des Industries Mécaniques, Senlis. 